\title{
激光在大气传输中方向起伏规律的探讨
}

\author{
戴 邦 雯 \\ (上海市地展办公室)
}

激光在湍流大气传输中, 直接影响激光方向起伏的因素, 主要是由于大气温度场的起伏而 引起的大气折射率随机场的效应.

大气折射率起伏场的存在，使激光在大气中传输方向发生随机的变化，因而产生光束漂 移、像点抖动等现象.

由于大气折射率场随机的变化，瞬时的空气层界面亦随机变化，但在相对静止的大气折射 率场中,空气层界面应近似地与地形起伏相一致,因为光线的折射,主要与温度梯度有关, 日间 受太阳照射, 地面温度比上空气层温度为高, 所以温度梯度为负, 即离地面愈高温度愈低. 夜 间则相反,温度梯度为正。

本文根据波长 $\lambda 、$ 温度 $T$ 、气压 $P$ 、视线与空气层界面及水准面夹角 $i 、 \theta$, 空气层界面与 水准面夹角 $\alpha$, 距离 $L$ 等因素,推导出方向起伏的大气效应计算公式

$$
h=f(\lambda, T, P 、 i, \theta 、 \alpha, L) .
$$

当视线从 0 空气层到 $n+1$ 空气层时，由于层之间空气密度不一致，于是光线就受到折 射, 当激光轴与水准轴的高度角为 $\theta$ 时, 在没有大气效应的情况下, 激光沿 $E C$ 直线前进, 激 光点应落在被观测标尺的 $C$ 点上, 在有大气效应的情况下, 激光点落在 $F$ 点, 大气效应 $h=$ $\sum_{j=0}^{n} \Delta_{i+1}$

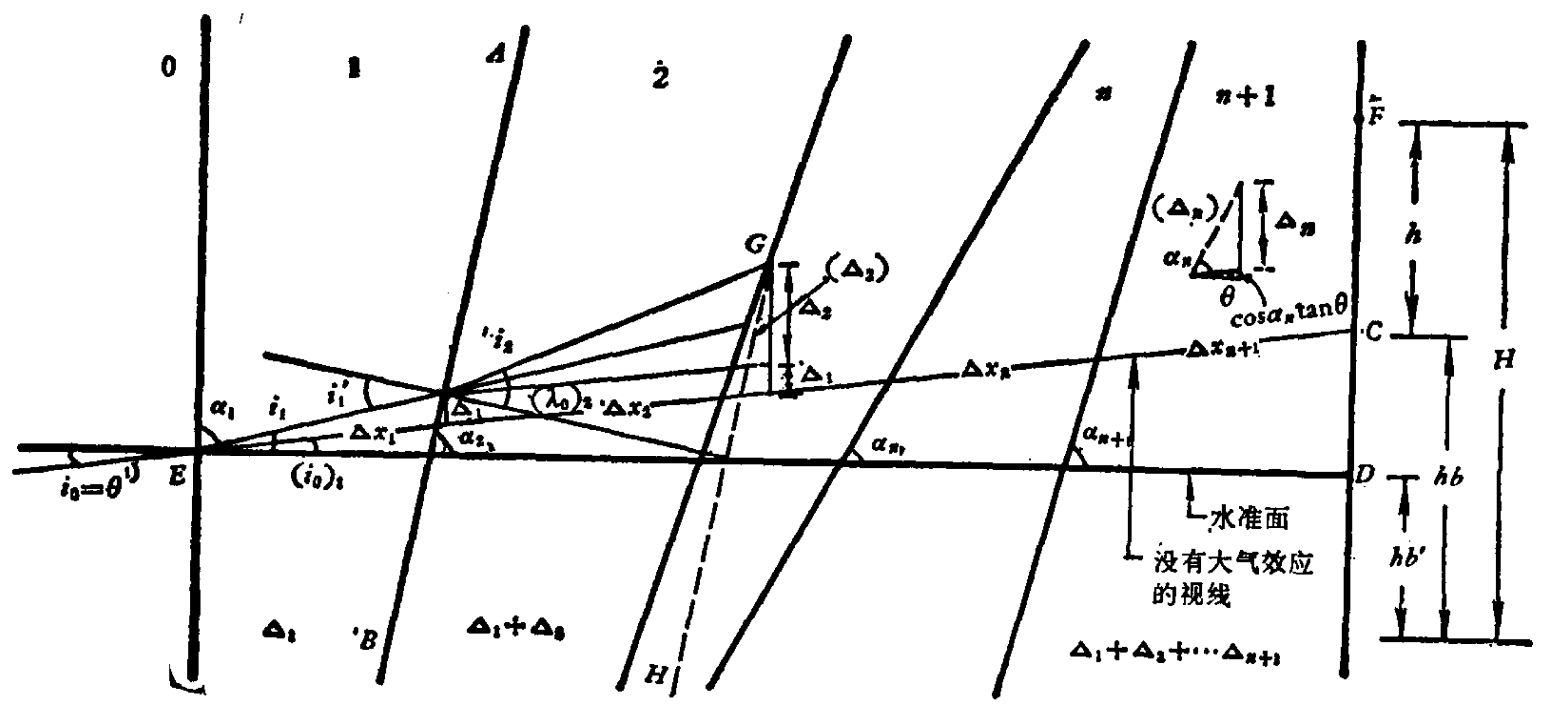

图 1

在大地水准测量中 $i_{0}=\theta$

本文 1978 年 6 月 13 日收到. 1979 年 2 月 13 日收到修改稿. 
视线的行程是一条空间曲线, 本文仅讨论垂直折射在大气传输中的规律, 当然所推导出的 公式对水平方向也一概适用.

视线通过的空气层界面是非常复杂的, 它与气象、地形起伏、覆被等因素有关,界面与界面 之间一般说应是不平行的, 而且是在变化的, 假定 $\alpha_{0}=\alpha_{1}, \alpha_{2}, \alpha_{3} \cdots \alpha_{n+1}$ 倾角不相等, 为了进 行运算, 从 $G$ 点引线 $G H / / A B$. 以下均依次类推,皆划引线与前一界面平行.

从图 1 可知, $i_{i}^{\prime}=i_{i}+\alpha_{i}-\alpha_{j+1},\left(i_{0}\right)_{j+1}=i_{0}+\alpha_{1}-\alpha_{i+1}$. $\left(\Delta_{i+1}\right)=\left(\tan i_{j+1}-\tan \left(i_{0}\right)_{j+1}\right)\left|\sin \left(\alpha_{i+1}-\theta\right)\right| \Delta x_{i+1}, \Delta_{i+1}=\left(\Delta_{i+1}\right)\left(\sin \alpha_{j+1}-\cos \alpha_{i+1} \tan \theta\right)$.

在标准状态下 ( $T=0^{\circ} \mathrm{C}, P=760$ 毫米承柱) 和干燥空气中时,群折射率 (波长 $\lambda$ 以微米 计)和波长的依赖关系为 ${ }^{[1]}$

$$
\eta_{0}=1+2.876 \times 10^{-4}+\frac{4.887 \times 10^{-6}}{\lambda^{2}}+\frac{6.80 \times 10^{-8}}{\lambda^{4}} .
$$

折射率 $\left(\eta_{i}\right)$ 与温度 $(T)$ 、压力 $(p)$ 湿度 $(e)$ 等参数关系表示为 ${ }^{[1]}$

$$
\eta_{j}=1+\frac{\eta_{0}-1}{1+\alpha T_{i}}\left(\frac{P_{j}}{760}\right)-\frac{5.5 \times 10^{-9} e}{\left(1+\alpha T_{j}\right)}, \alpha=0.00367 \text {. }
$$

斯涅耳定律

$$
\eta_{i} \sin i_{j}=\eta_{i+1} \sin i_{i+1} \text {. }
$$

双波长激光器发射出的两种不同波长的激光束, 则对于第一种波长可得

$$
\begin{aligned}
h_{1} & =\int_{0}^{L} f_{1}(x) d x=\lim _{\substack{n \rightarrow \infty \\
\Delta x \rightarrow 0}} \sum_{j=0}^{n} \Delta_{1 \cdot j+1}=\lim _{\substack{n \rightarrow \infty \\
\Delta x \rightarrow 0}} \sum_{j=0}^{n}\left[\left(\tan i_{1 \cdot j+1}-\tan \left(i_{1 \cdot 0}\right)_{j+1}\right)\right. \\
& \left.\times\left(\sin \alpha_{j+1}-\cos \alpha_{1+1} \tan \theta\right) \cdot\left|\sin \left(\alpha_{j+1}-\theta\right)\right| \cdot \Delta x_{j+1}\right] .
\end{aligned}
$$

对于第二种波长可得

$$
\begin{aligned}
& h_{2}=\int_{0}^{L} f_{2}(x) d x=\lim _{\substack{n \rightarrow \infty \\
\Delta x \rightarrow 0}} \sum_{j=0}^{n} \Delta_{2 \cdot j+1}=\lim _{\substack{n \rightarrow \infty \\
\Delta x \rightarrow 0}} \sum_{j=0}^{n}\left[\left(\tan i_{2 \cdot j+1}-\tan \left(i_{2 \cdot 0}\right)_{j+1}\right)\right. \\
& \left.\times\left(\sin \alpha_{j+1}-\cos \alpha_{j+1} \tan \theta\right) \cdot\left|\sin \left(\alpha_{j+1}-\theta\right)\right| \cdot \Delta x_{j+1}\right] .
\end{aligned}
$$

为了比较 $h_{1} 、 h_{2}$ 之间的关系, 我们假定空气层界面完全平行 (即 $\alpha$ 相等), 且空气层间隔 $\Delta x$ 也相等, 这时

$$
i_{j}^{\prime}=i_{j},\left(i_{0}\right)_{j+1}=i_{0}, \eta_{0} \sin i_{0}=\eta_{1} \sin i_{1}=\cdots=\eta_{n+1} \sin i_{n+1} .
$$

同时, (7)、(8) 式可写成

$$
\begin{aligned}
& h_{1}=\int_{0}^{L} f_{3}(x) d x=\lim _{\substack{n \rightarrow \infty \\
\Delta x \rightarrow 0}}\left[\sum_{j=0}^{n}\left(\tan i_{1 \cdot j+1}-\tan i_{1 \cdot 0}\right)\right](\sin \alpha-\cos \alpha \tan \theta) \cdot|\sin (\alpha-\theta)| \Delta x . \\
& h_{2}=\int_{0}^{L} f_{4}(x) d x=\lim _{\substack{n \rightarrow \infty \\
\Delta x \rightarrow 0}}\left[\sum_{j=0}^{n}\left(\tan i_{2 \cdot j+1}-\tan i_{2 \cdot 0}\right)\right](\sin \alpha-\cos \alpha \tan \theta) \cdot|\sin (\alpha-\theta)| \Delta x .
\end{aligned}
$$

将 (10)、(11) 式进一步展开, 并将 (4)、(5) 代入略去湿度的影响, 可得

$$
\begin{aligned}
h_{1} & =\left(\eta_{01}-1\right)\left\{\sum _ { j = 0 } ^ { n } ( \frac { 1 } { 1 + \alpha T _ { 0 } } \frac { P _ { 0 } } { 7 6 0 } - \frac { 1 } { 1 + \alpha T _ { i + 1 } } \frac { P _ { j + 1 } } { 7 6 0 } ) \frac { \operatorname { s i n } i _ { 1 \cdot 0 } } { \eta _ { 1 \cdot j + 1 } } \left[1+\frac{1}{2}\left(\eta_{1 \cdot 0}^{2}+\eta_{1 \cdot 0} \eta_{1 \cdot j+1}\right.\right.\right. \\
& \left.\left.\left.+\eta_{1 \cdot j+1}^{2}\right) \frac{\sin ^{2} i_{1 \cdot 0}}{\eta_{1 \cdot j+1}^{2}}+\cdots\right](\sin \alpha-\cos \alpha \tan \theta) \cdot|\sin (\alpha-\theta)| \Delta x\right\}=\left(\eta_{01}-1\right) w_{1} .
\end{aligned}
$$




$$
\begin{aligned}
h_{2} & =\left(\eta_{02}-1\right)\left\{\sum _ { j = 0 } ^ { \infty } ( \frac { 1 } { 1 + \alpha T _ { 0 } } \frac { P _ { 0 } } { 7 6 0 } - \frac { 1 } { 1 + \alpha T _ { i + 1 } } \frac { P _ { i + 1 } } { 7 6 0 } ) \frac { \operatorname { s i n } i _ { 2 \cdot 0 } } { \eta _ { 2 \cdot ; + 1 } } \left[1+\frac{1}{2}\left(\eta_{2 \cdot 0}^{2}+\eta_{2 \cdot 0} \eta_{2 \cdot \cdot+1}\right.\right.\right. \\
& \left.\left.\left.+\eta_{2 \cdot \cdot+1}^{2}\right) \frac{\sin ^{2} i_{2 \cdot 0}}{\eta_{2 \cdot ;+1}^{2}}+\cdots\right](\sin \alpha-\cos \alpha \tan \theta) \cdot|\sin (\alpha-\theta)| \Delta x\right\}=\left(\eta_{02}-1\right) w_{2 \bullet} .
\end{aligned}
$$

比较 (12)、(13) 式, 可知在一定的精度要求下

$$
r=\gamma=\frac{h_{1}}{h_{2}}=\frac{\eta_{01}-1}{\eta_{02}-1}=\text { 对于任何两个给定的波长是常数. }
$$

因为:（1）使用双波长激光器时的起始状态相同, 即 $i_{1 \cdot 0}=i_{2 \cdot 0}$;

(2) $\frac{\sin i_{1 \cdot 0}}{\eta_{1 \cdot i+1}} 、 \frac{\sin i_{2 \cdot 0}}{\eta_{2 \cdot i+1}}$ 都小于 1 , 所以此级数是收玫的;

（3）气象条件及空气层界面变化情况相同;

(4) $\eta_{1 \cdot j+1} 、 \eta_{2 \cdot j+1} 、 \frac{\eta_{1 \cdot j+1}}{\eta_{2 \cdot j+1}}$ 都接近于 $1, \beta=\frac{w_{1}}{w_{2}}$ 也趋近于 1 .

(7)、(8) 式不能直接写出式 (12)、(13) 的简单形式，但总可化为正弦函数的复合函数， 最后仍可得到 (14) 式的结果.

曾假定各种不同的气象因素, 空气层界面的变化及忽略湿度和考虑湿度影响情况下，用 (7)、(8) 式进行了大量的电算工作.

忽略湿度的影响, $\gamma=\gamma_{1} \cdot \beta$ 与 $\gamma=\frac{\eta_{01}-1}{\eta_{02}-1}$ 相差仅 $10^{-6}$ 数量级, 即 $\beta=1 \pm 10^{-6}$.

当在温度 $40^{\circ} \mathrm{C}$ 的饱和水蒸汽压 55.35 毫米情况下, $\gamma$ 与 $\gamma$ 相差 $10^{-5}$ 数量级, 即 $\beta=1 \pm$ $10^{-5}$, 但用观测站的湿度代入可修正 $\gamma$ 㴗, 将能提高 $\gamma$ 的精度.

通过大量的电算, 所获得的结果是令人满意的, 它表明本文主要的论证, 公式 (14) 是存在 的, 具有实际价值的.

若研制双波长激光自动安平水准仪, 进行水准测量时 (图 2), 从测量得到:

$$
\delta=H_{2}-H_{1}=h_{2}-h_{1} \text {. }
$$

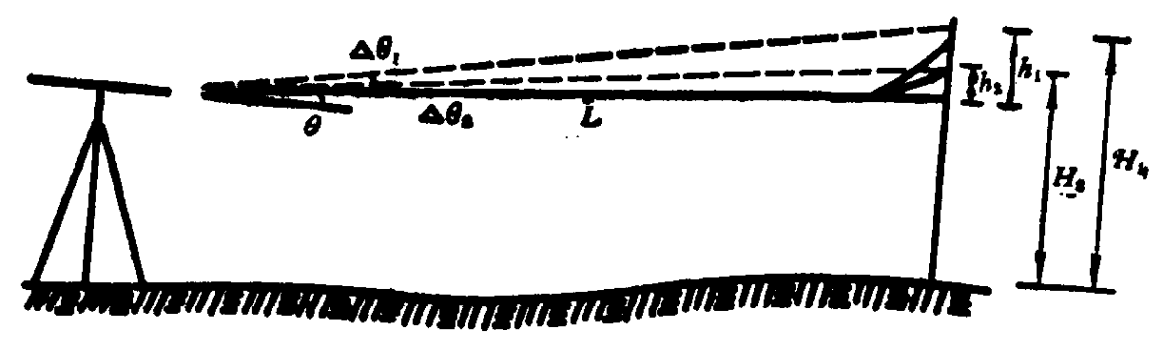

图 2

据据 (14)、(15) 式可得

$$
h_{2}=\frac{\delta}{1-\gamma}=\frac{\eta_{02}-1}{\eta_{02}-\eta_{01}} \delta .
$$

若需将线段量大气效应 $h_{1} 、 h_{2}$ 化为角色散值 $\Delta \theta_{1} 、 \Delta \theta_{2}$ (图 2), 则可表达为

$$
\Delta \theta_{1}=\frac{\left(\eta_{01}-1\right) \cdot \cos \theta}{L} \cdot w_{1}, \quad \Delta \theta_{2}=\frac{\left(\eta_{02}-1\right) \cdot \cos \theta}{L} \cdot w_{2 .}
$$

同样可在一定精度要求下得 


$$
\gamma=\frac{\Delta \theta_{1}}{\Delta \theta_{2}}=\frac{\eta_{01}-1}{\eta_{02}-1}=\text { 对于任何两个给定的波长是常数. }
$$

在国内外, 关于激光在大气传输方向起伏大气效应问题, 开展了广泛的理论和实验研究, 本文仅就以下几个问题进行了评价和讨论.

1. Hodara ${ }^{[2]}$ 设想光线通过的介质按相关距离 $L_{c}$ 分层, 相邻两层的折射率相差 $\Delta n$ （图 3).

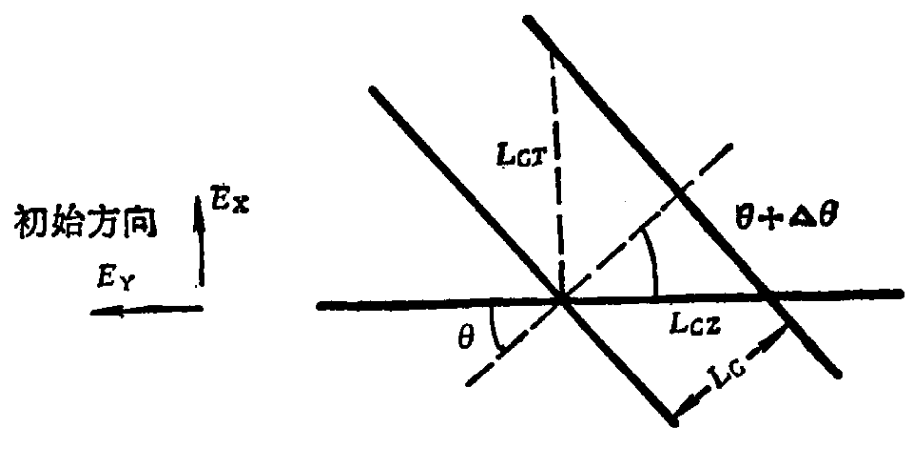

图 3

$$
\Delta \theta_{C}=\Delta n \tan \theta=\Delta n \frac{L_{C Z}}{L_{C T}}
$$

若光程长为 $L$, 光束将通过 $L / L_{C z}$ 个湍流体, 则到达角起伏方差 $\sigma_{\theta}^{2}$ 为:

$$
\sigma_{\theta}^{2}=\left\langle\Delta \theta^{2}\right\rangle=\Sigma \overline{\Delta \theta}_{C}^{i}=\frac{L}{L_{C Z}} \overline{\Delta n^{2}}\left(\frac{L_{C Z}}{L_{C T}}\right)^{2}=\overline{\Delta n^{2}} \frac{L_{C Z} L}{L_{C T}^{2}} .
$$

Hodara 举例取 $L_{C Z} \approx 30 \mathrm{M}, L_{C T} \approx 1 \mathrm{M}, \sqrt{\overline{\Delta n^{2}}}=10^{-6}$, 当传输 30 公里, 可算得 $\Delta \theta \cong 1$ 毫弧度.

与本文 (7)、(8) 式比较, 可知 Hodara 公式有几个问题:

(1) 未考虑高度角 $\theta$ (即天顶距 $Z=90^{\circ}-\theta$ ) 的影响;

（2）光束通过的湍流体空气层界面瞬时变化的因素在式中未能加以反映.

2. Bender 的大气折射率改正公式 ${ }^{[1]}$ 的讨论

$$
\Delta s=s_{2}-s_{1}=A s_{2}, A=\left(\frac{\eta_{2}-\eta_{1}}{\eta_{2}-1}\right)=\left(\frac{\eta_{02}-\eta_{01}}{\eta_{02}-1}\right)=\text { 对于任何两个给定的波长是 }
$$
常数。

（1）分析式 (21), $A=\frac{\eta_{02}-\eta_{01}}{\eta_{02}-1}=1-\frac{\eta_{01}-1}{\eta_{02}-1}=1-\gamma, s_{2}=\frac{\Delta s}{1-\gamma}$.

(2) 比较 (14)、(22) 两式, 所得结果形式相同. 显然, Bender 公式中 $A$ 的出现，主要由 于 $\gamma=\frac{s_{1}}{s_{2}}=\frac{\eta_{01}-1}{\eta_{02}-1}$ 而导致.

3. 上面曾提及相对静止的大气折射率起伏场, 应大致与地形起伏相一致, 所以当 $\theta$ 角逐渐 增大时, $i$ 角则逐渐减小, 从 (17) 式可知 $\Delta \theta$ 随 $i$ 角减小 $\theta$ 角增大而减少, 这就从理论上说 明, 为什么斜程传输时, 无论是地对空或空对地, 到达角起伏比相当距离上的水平传输时要小 的原因. 上海天文台观测到星光抖动角一般在 5 微弧度以下，在天顶处抖动的均方根年平均 值为 1.5 微弧度, 从公式 (17) 亦可说明天顶星光抖动角应为最小. 
4. 将 (16) 式 $h_{2}=\frac{\delta}{1-\gamma}$ 全微分可得

$$
d h_{2}=\delta(1-\gamma)^{-2} d \gamma+(1-\gamma)^{-1} d \delta .
$$

根据误差传播规律得到: $M_{h_{2}}=\sqrt{\left[\delta(1-\gamma)^{-2}\right]^{2} m_{\gamma}^{2}+\left[(1-\gamma)^{-1}\right]^{2} m_{\delta}^{2}}$.

由 (24) 式可知, 研制多波长激光的光学仪器, 光电接受装置灵敏度 $\left(m_{\delta}\right)$ 愈高, $\gamma$ 的精度 $\left(m_{r}\right)$ 愈高, $\gamma$ 愈大(在避开反常色散区情况下, 第一种波长愈短, 第二种波长愈长 $\gamma$ 愈大), 则 仪器的测量精度愈高.

5. 大地测量学者对大地水准测量的折光误差是偶然性还是系统性误差尚有争 ${ }^{\text {议 }}{ }^{[3]}$ ，我们 认为由于观测方向 (距离相等)的气象、地形起伏、覆被等因素的不同，一般沿倾斜的地面上进 行水准测量时, 折光误差是系统性的, 但 Lallemand、Павлов 等推导的折光误差公式, 至今未 能用于生产上 ${ }^{[4]}$, 是因为未考虑 $i$ 角及其变化因素的原因.

致谢: 吕建华同志曾对本文提了不少宝贵意见，电算的程序设计由仇志荣同志协助完成,均在此表示感 谢.

\title{
考文蝴
}

[1] Bender, R. A., Earthquake prediction from laser surveying, A REPORT, (NASA SP-5042, 27-28).

「2] Hodara, H., Laser ware propagation through the atmosphere, Proc IEEE, 54 (1966), 368-375.

[3] Энтин И. И., 高精度水准测最, 测绘出版社, 1958, 35-38.

[4] 武汉测绘学院大地测量教研组, 大地测量学(上册), 中国工业出版社, 1964, 301-302.

\section{DISCUSSION ON DIRECTIONAL FLUCTUATION LAW OF LASER-BEAM PROPAGATION THROUGH THE ATMOSPHERE}

\section{DaI Bang-wen （戴邦要）}

\begin{abstract}
In this paper the course of line-of-sight travel is considered as passing through an unlimited number of mutually unparallelled interfaces of the atmosphere. According to the principle of light refraction in different media, such as air, the formula of the directional fluctuation law of laser-beam propagation through the turbulent atmosphere is derived by opticogeometrical method.

In the course of derivation, it was found that for the two laser-beams of different wavelengths transmitted by dual-wavelength laser in the same optical system, $\gamma=\frac{h_{1}}{h_{2}}=\frac{\eta_{01}-1}{\eta_{02}-1}$ is a constant for any two given wavelengths within the specific requirements of accuracy.
\end{abstract}

